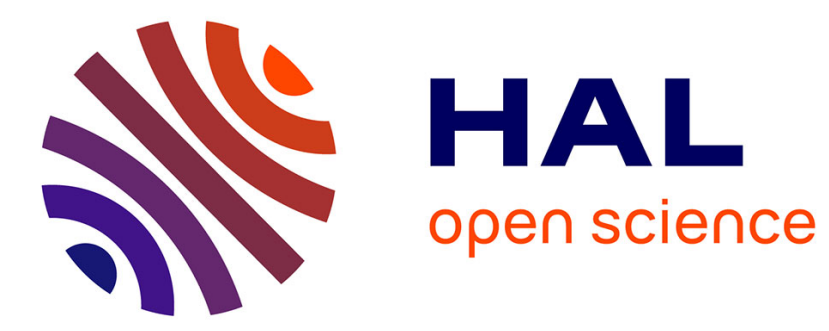

\title{
Global Gene Regulation in Metabolic Networks
}

Diego A. Oyarzun, Madalena Chaves

\section{To cite this version:}

Diego A. Oyarzun, Madalena Chaves. Global Gene Regulation in Metabolic Networks. [Research Report] RR-7468, INRIA. 2010, pp.15. inria-00541112

\section{HAL Id: inria-00541112 \\ https://hal.inria.fr/inria-00541112}

Submitted on 29 Nov 2010

HAL is a multi-disciplinary open access archive for the deposit and dissemination of scientific research documents, whether they are published or not. The documents may come from teaching and research institutions in France or abroad, or from public or private research centers.
L'archive ouverte pluridisciplinaire HAL, est destinée au dépôt et à la diffusion de documents scientifiques de niveau recherche, publiés ou non, émanant des établissements d'enseignement et de recherche français ou étrangers, des laboratoires publics ou privés. 
INSTITUT NATIONAL DE RECHERCHE EN INFORMATIQUE ET EN AUTOMATIQUE

\title{
Global Gene Regulation in Metabolic Networks
}

\author{
Diego A. Oyarzún - Madalena Chaves
}

\section{$\mathbf{N}^{\circ} 7468$}

November 2010

\section{apport}

derecherche 



\title{
Global Gene Regulation in Metabolic Networks
}

\author{
Diego A. Oyarzún* Madalena Chavesł \\ Thème : Observation, modélisation et commande pour le vivant \\ Équipe-Projet Comore \\ Rapport de recherche $\mathrm{n}^{\circ} 7468$ - November 2010 - 15 pages
}

\begin{abstract}
Genetic feedback is one of the mechanisms that enables metabolic adaptations to environmental changes. The stable equilibria of these feedback circuits determine the observable metabolic phenotypes. We consider an unbranched metabolic network with one metabolite acting as a global regulator of enzyme expression. Under switch-like regulation and exploiting the time scale separation between metabolic and genetic dynamics, we develop geometric criteria to characterize the equilibria of a given network. These results can be used to detect mono- and bistability in terms of the gene regulation parameters for any combination of activation and repression loops.
\end{abstract}

Key-words: kinetic modeling and control of biological systems, metabolic networks, genetic networks

The first author was supported by a NBIPI Career Enhancement and Mobility Fellowship (co-funded by Marie Curie Actions, the Irish Higher Education Authority Programme for Third Level Institutions Cycle 4 and the Italian National Research Council). The second author was supported in part by the INRIA-INSERM project ColAge.

* Hamilton Institute, National University of Ireland, Maynooth, Co. Kildare, Ireland (email: diego.oyarzun@nuim.ie).

${ }^{\dagger}$ COMORE, INRIA Sophia Antipolis, 2004 route des Lucioles, BP 93, 06902 Sophia Antipolis, France (e-mail: madalena.chaves@inria.fr)

Centre de recherche INRIA Sophia Antipolis - Méditerranée 2004, route des Lucioles, BP 93, 06902 Sophia Antipolis Cedex Téléphone : +334923877 77 — Télécopie : +33492387765 


\section{Régulation Génétique Globale de Réseaux Métaboliques}

Résumé : Les réactions métaboliques sont régulées par des enzymes mais, en même temps, les gènes codant pour ces enzymes peuvent être régulées par les produits des réseaux métaboliques. Ce mécanisme de régulation en boucle fermée permet l'adaptation d'un système métabolique aux changements environnementaux. Nous étudions un système composé d'une chaine métabolique couplée à un réseau génétique, où un des métabolites joue le rôle de régulateur global de l'expression enzymatique. Le métabolite régulateur peut être soit répresseur soit activateur de chaque gène. Sous l'hypothèse que l'échelle de temps du système métabolique est plus rapide que celle du réseau génétique, le système composé peut être simplifié. Dans ce cas, nous développons des critères géométriques pour caractériser les équilibres du système et analyser la monoou bi- stabilité du système en termes des paramètres.

Mots-clés : modélisation et contrôle de systèmes biologiques, réseaux métaboliques, réseaux génétiques 


\section{Introduction}

Metabolism and gene expression are two fundamental levels of cellular regulation. Gene expression can impact metabolic levels through changes in enzyme concentration and, conversely, metabolic species can influence gene transcription and hence modulate enzyme synthesis 9 . These two levels have specific functions and properties, and it remains a challenge to characterize the interconnections between them. The configurations of metabolic-genetic interactions can lead to a diverse range of dynamic behaviors, each one of which defines a specific metabolic phenotype. Our understanding of natural regulatory circuits is important not only for revealing the design principles that underlie observed metabolic dynamics, but also for our ability to design synthetic circuits that enable new phenotypes [1].

A specific phenotype depends, among others, on: the regulatory topology (i.e. which metabolites regulate which enzymes), logic (i.e. activation or repression), and the sensitivity of regulation (i.e. graded or switch-like regulation). In this paper we present a mathematical analysis of these properties by studying the interconnection between an unbranched metabolic network and a one-to-all genetic control circuit. The gene circuit implements a form of global control in the sense that one metabolic species modulates the activity of all enzymes.

The model integrates the classical kinetic equations to represent metabolite dynamics and piecewise affine (PA) systems to represent gene regulation (Section 22. To analyze the model we consider that metabolic reactions happen in a much faster time scale when compared to gene transcription or translation [1. PA models 2] provide a convenient way of encoding switch-like regulation with relatively few parameters (only expression rates and regulatory thresholds), whereas the time scale separation allows for an approximation of the metabolic dynamics by a system of algebraic equations. This approximation ultimately leads to a reduction of the model to a 2-dimensional PA system defined in conic domains (Section 3). Solutions of these type of systems can be obtained by defining the system as a differential inclusion and using a construction due to [5]. The conic geometry introduces new features in the solutions at the switching domains, and we characterize the existence and stability of the possible sliding motion and singular equilibria (Section 4). This analysis is applied to study a particular configuration of the globally regulated metabolic chain (Section 5).

\section{Global genetic control}

We consider an unbranched metabolic network with one metabolite as a global regulator of enzyme expression. A schematic diagram of such class of networks is shown in Figure 1 where $s_{i}$ denotes the concentration of the $i^{\text {th }}$ metabolite and $v_{i}$ is the rate of the $i^{\text {th }}$ reaction (catalyzed by an enzyme with concentration $\left.e_{i}\right)$.

As a way of accounting for the mass exchange between the network and its environment, we assume that the metabolic substrate $s_{0}$ is constant. For the sake of generality, in this paper we deal with networks of $n$ metabolites and $n+1$ enzymes regulated by a single global regulator $s_{\ell-1}(\ell>1)$. The rate of 


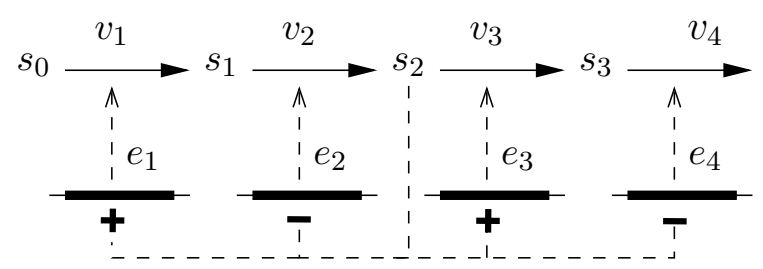

Figure 1: Global gene regulation for an unbranched network.

change of both metabolite and enzyme concentrations can be described by

$$
\begin{aligned}
\dot{s}_{i} & =v_{i}\left(s_{i-1}, e_{i}\right)-v_{i+1}\left(s_{i}, e_{i+1}\right), \\
\dot{e}_{i} & =\kappa_{i}^{0}+\kappa_{i}^{1} \sigma_{i}\left(s_{\ell-1}, \theta_{i}\right)-\gamma_{i} e_{i} .
\end{aligned}
$$

where $\kappa_{i}^{0}, \kappa_{i}^{1}, \theta_{i}, \gamma_{i}$ are positive parameters. The metabolic model (1) arises from the mass balance between the reactions that produce and consume $s_{i}$, whereas the model for the enzyme concentrations (2) comes from the balance between protein synthesis and degradation (modeled as a linear process). The constant $\kappa_{i}^{0}$ represents a basal expression level of protein $e_{i}$, whereas $\kappa_{i}^{1}$ and the functions $\sigma_{i}$ model the effect of the global regulator on the synthesis rates.

The regulatory function $\sigma_{i}\left(s_{\ell-1}, \theta_{i}\right)$ represents the lumped effect of gene expression control by a transcription factor, together with its interaction with the regulator $s_{\ell-1}$. To account for the typical switch-like nature of transcriptional regulation, $\sigma_{i}(x, \theta)$ is assumed to be a step function; depending on whether gene expression is activated or repressed by $s_{\ell-1}$, we assign $\sigma_{i}=\sigma^{+}$or $\sigma_{i}=\sigma^{-}=$ $1-\sigma^{+}$, respectively, with

$$
\sigma^{+}(x, \theta)=\left\{\begin{array}{ll}
0, & x<\theta \\
1, & x>\theta
\end{array} .\right.
$$

This class of regulatory functions is widely used used in the analysis of genetic networks [10, 3] and was first suggested in [6. In the sequel we will not presuppose a specific form of the enzyme kinetics; instead to keep the analysis as general as possible, we make the following assumption on the enzyme kinetics. Assumption 1. The metabolic reaction rates are linear in the enzyme concentrations and non-decreasing functions of the metabolite concentrations, so that the rate functions are written as

$$
v_{i}\left(s_{i-1}, e_{i}\right)=g_{i}\left(s_{i-1}\right) e_{i},
$$

where $g_{i}$ is the enzyme turnover rate (i.e. the reaction rate per unit of enzyme concentration) and satifies

$$
\frac{\partial g_{i}\left(s_{i-1}\right)}{\partial s_{i-1}} \geq 0 .
$$

The monotonicity condition in (5) accounts for a broad class of saturable enzyme kinetics that includes, in particular, Michaelis-Menten and Hill kinetics [4. In the rest of the paper we aim at characterizing the equilibria of the feedback system in (1) $-(2)$. 


\section{Time scale separation}

\subsection{Quasi steady state approximation}

Metabolic dynamics operate in a much shorter time scale than their genetic counterpart [1. This property allows the approximation of the nonlinear dynamics in (1) by an algebraic relationship between the enzymes and metabolite concentrations. If the metabolites are assumed to be in quasi steady state (QSS) with respect to the enzyme concentrations, then we set $\dot{s}_{i}(t)=0$ for all $t \geq 0$ to obtain

$$
g_{i+1}\left(s_{i}(t)\right)=g_{i}\left(s_{i-1}(t)\right) \frac{e_{i}(t)}{e_{i+1}(t)}
$$

Equation (6) holds for every $i=1,2, \ldots, n$ and hence it is equivalent to

$$
g_{i}\left(s_{i-1}(t)\right)=g_{1}\left(s_{0}\right) \frac{e_{1}(t)}{e_{i}(t)}
$$

The trajectory of the global regulator can be then computed by solving the equation

$$
g_{\ell}\left(s_{\ell-1}(t)\right)=g_{1}\left(s_{0}\right) \frac{e_{1}(t)}{e_{\ell}(t)}
$$

If the function $g_{i}$ does not saturate, a nonnegative solution of (7) exists at all times. However, for saturable functions one must guarantee that this is true by stating appropriate assumptions on the parameters (for instance, of the form $\left.\kappa_{i}^{0} / \gamma_{i} \geq\left(g_{1}\left(s_{0}\right) / \max \left(g_{i}\right)\right)\left(\kappa_{1}^{0}+\kappa_{1}^{1}\right) / \gamma_{1}\right)$. For space reasons we will assume that solutions of (7) do exist and omit the details. A key aspect of this approximation is that the solution of (1)-2 depends only on two proteins. The dynamics of the complete feedback system can thus be fully characterized by analyzing the 2-dimensional phase plane of the differential equations

$$
\begin{aligned}
& \dot{e}_{1}=\kappa_{1}^{0}+\kappa_{1}^{1} \sigma_{1}\left(s_{\ell-1}, \theta_{1}\right)-\gamma_{1} e_{1}, \\
& \dot{e}_{\ell}=\kappa_{\ell}^{0}+\kappa_{\ell}^{1} \sigma_{\ell}\left(s_{\ell-1}, \theta_{\ell}\right)-\gamma_{\ell} e_{\ell},
\end{aligned}
$$

subject to $s_{\ell-1}$ satisfying (8).

\subsection{Equivalent piecewise affine system in conic domains}

The algebraic equation in (8) can be interpreted as a mapping from $\mathbb{R}_{\geq 0}$ to $\mathbb{R}_{\geq 0}^{2}$, whereby each value of the regulator $s_{\ell-1}$ maps into a half-line in the $\left(e_{1}, e_{\ell}\right)$ plane. Moreover, as a consequence of the monotonicity of $g_{\ell}$, the partition of $\mathbb{R}_{\geq 0}$ induced by the thresholds can be mapped into a partition of $\mathbb{R}_{\geq 0}^{2}$ : if $s_{\ell-1}<\theta_{i}$ then

$$
g_{\ell}\left(s_{\ell-1}\right)<g_{\ell}\left(\theta_{i}\right)
$$

which combined with (8) yields

$$
e_{\ell}>\beta_{i} e_{1},
$$


with $\beta_{i}=g_{1}\left(s_{0}\right) / g_{\ell}\left(\theta_{i}\right)$. The relation in (11) defines a cone in the $x=\left(e_{1}, e_{\ell}\right)$ plane

$$
D_{i}=\left\{x \in \mathbb{R}_{\geq 0}^{2}: x_{2}>\beta_{i} x_{1}\right\},
$$

and we define its complementary cone as $\bar{D}_{i}=\mathbb{R}_{\geq 0}^{2} \backslash\left(D_{i} \cup S_{i}\right)$ with $S_{i}$ the half-line

$$
S_{i}=\left\{x \in \mathbb{R}_{\geq 0}^{2}: x_{2}=\beta_{i} x_{1}\right\} .
$$

The half-line $S_{i}$ is a subset of the $\left(e_{1}, e_{\ell}\right)$ plane where the regulator reaches the switching threshold $\theta_{i}$. The dynamics of the reduced system in (9) depend on the value of $s_{\ell-1}$ with respect to the thresholds $\theta_{1}$ and $\theta_{\ell}$. Assume, without loss of generality, that $\theta_{1}<\theta_{\ell}$ (the problem can be treated analogously in the case $\theta_{1}>\theta_{\ell}$, and the case $\theta_{1}=\theta_{\ell}$ is treated as in Section 4). With the previous definitions we can establish the following relations

$$
\begin{aligned}
s_{\ell-1}<\theta_{1} & \Longleftrightarrow x \in R_{1}, \\
\theta_{1}<s_{\ell-1}<\theta_{\ell} & \Longleftrightarrow x \in R_{1 \ell}, \\
s_{\ell-1}>\theta_{\ell} & \Longleftrightarrow x \in R_{\ell},
\end{aligned}
$$

where $R_{1}=D_{1}, R_{1 \ell}=\bar{D}_{1} \cap D_{\ell}$ and $R_{\ell}=\bar{D}_{\ell}$. In the sequel we refer to $S_{i}$ as a switching domain, whereas the cones $R_{j}$ are called regular domains (see [2] for detailed definitions). The system in (9) is equivalent to a piecewise affine (PA) system [8] in conic domains

$$
\dot{x}=h(x)-\Gamma x,
$$

where

$$
h(x)=\left\{\begin{array}{ll}
h^{1} & x \in R_{1} \\
h^{1 \ell} & x \in R_{1 \ell} \\
h^{\ell} & x \in R_{\ell}
\end{array} \quad \quad \quad=\left[\begin{array}{cc}
\gamma_{1} & 0 \\
0 & \gamma_{\ell}
\end{array}\right] .\right.
$$

The vectors $h^{1}, h^{1 \ell}$ and $h^{\ell}$ are constant and their values depend on whether $s_{\ell-1}$ activates or represses the expression of enzymes $e_{1}$ and $e_{\ell}$. For example, in the case of repression (i.e. $\sigma_{1}=\sigma^{-}$and $\sigma_{\ell}=\sigma^{-}$) we have

$$
h^{1}=\left[\begin{array}{l}
\kappa_{1}^{0}+\kappa_{1}^{1} \\
\kappa_{\ell}^{0}+\kappa_{\ell}^{1}
\end{array}\right], \quad h^{1 \ell}=\left[\begin{array}{c}
\kappa_{1}^{0} \\
\kappa_{\ell}^{0}+\kappa_{\ell}^{1}
\end{array}\right], \quad h^{\ell}=\left[\begin{array}{c}
\kappa_{1}^{0} \\
\kappa_{\ell}^{0}
\end{array}\right] .
$$

These vectors determine the location of the focal points of the PA system, defined as

$$
\phi^{1}=\Gamma^{-1} h^{1}, \quad \phi^{1 \ell}=\Gamma^{-1} h^{1 \ell}, \quad \phi^{\ell}=\Gamma^{-1} h^{\ell} .
$$

The conic partition of the $\left(e_{1}, e_{\ell}\right)$ phase plane and the focal points are shown in Figure 2. For any $x\left(t_{0}\right)$ in a regular domain, e.g. $x\left(t_{0}\right) \in R_{1}$, the right-hand side of 20 is well defined and its solution satisfies a standard affine differential equation, that is

$$
x(t)=\phi^{1}+e^{\Gamma\left(t_{0}-t\right)}\left(x\left(t_{0}\right)-\phi^{1}\right), t \geq t_{0}
$$

so that $x(t)$ monotonically approaches $\phi^{1}$, possibly reaching the switching domain $S_{1}$, where the vector field of $(20)$ is not defined, and thus a specialized analysis is required. As we shall see in the next section, the location of the focal points plays a major role in the dynamics of 15 . 


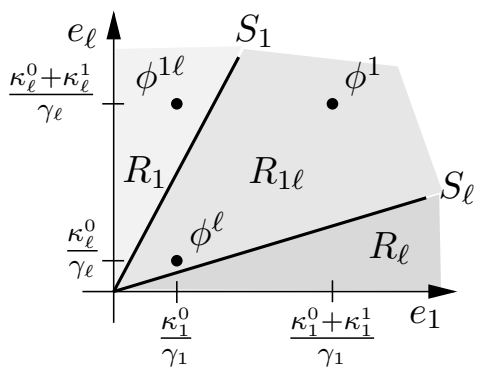

Figure 2: Conic partition of the state space and focal points when both $e_{1}$ and $e_{\ell}$ are repressed by metabolite $s_{\ell-1}$.

\section{Piecewise affine systems in cones}

To understand the dynamics of the PA system in (15), in this section we first study the solutions of a 2-dimensional PA system defined only in two cones (as opposed to three cones, cf. Figure 2). Consider the half-line $S=$ $\left\{x \in \mathbb{R}_{\geq 0}^{2}: x_{2}=\beta x_{1}\right\}$ with $\beta>0$, and the PA system

$$
\dot{x}=\left\{\begin{array}{ll}
f(x) & x \in D_{f} \\
g(x) & x \in D_{g}
\end{array},\right.
$$

where $D_{f}=\left\{x \in \mathbb{R}_{\geq 0}^{2}: x_{2}>\beta x_{1}\right\}$ and $D_{g}=\mathbb{R}_{\geq 0}^{2} \backslash\left(S \cup D_{f}\right)$. We denote the generating vector of $S$ as $\eta=\left[\begin{array}{ll}1 & \beta\end{array}\right]^{T}$; the vector fields $f(x)=\left[\begin{array}{ll}f_{1}(x) & f_{2}(x)\end{array}\right]^{T}$ and $g(x)=\left[\begin{array}{ll}f_{1}(x) & f_{2}(x)\end{array}\right]^{T}$ are affine and given by

$$
f(x)=h^{f}-\Gamma x, \quad g(x)=h^{g}-\Gamma x,
$$

where $h^{f}$ and $h^{g}$ are entrywise positive vectors, and $\Gamma=\operatorname{diag}\left\{\gamma_{1}, \gamma_{2}\right\}>0$. The focal points of $f(x)$ and $g(x)$ are given by $\phi^{f}=\Gamma^{-1} h^{f}$ and $\phi^{g}=\Gamma^{-1} h^{g}$, respectively, and are assumed to satisfy $\phi^{f}, \phi^{g} \notin S$. Solutions of differential equations with discontinuous vector fields are typically characterized with a construction due to Filippov [5]. This method proceeds by extending 20 to a differential inclusion

$$
\dot{x} \in H(x), \forall x \in S,
$$

where $H(x)$ is a set-valued function defined as the closed convex hull of $f(x)$ and $g(x)$, i.e.

$$
H(x)=\left\{z \in \mathbb{R}^{2}: z=\alpha f(x)+(1-\alpha) g(x), 0 \leq \alpha \leq 1\right\} .
$$

The solutions of $(22)$ are understood in the following sense (see also [7, 2, for more details).

Definition 4.1 or a given $\rho_{0}$, a solution of $(22)$ in $[0, T]$ is an absolutely continuous function $\rho:[0, T] \rightarrow \mathbb{R}_{\geq 0}^{2}$ such that $\rho(0)=\rho_{0}$ and $\dot{\rho}(t) \in H(\rho(t))$ for almost all $t \in[0, T]$. 


\subsection{Solutions in the switching domain}

Depending on the directions of the vector fields $f(x)$ and $g(x)$, Filippov's construction may not allow for uniqueness of solutions in the switching domains [5]. When uniqueness can be guaranteed, then solutions of 22 can: (a) cross to a regular domain or, (b) slide along the switching surface $S$. Roughly speaking, case (a) occurs when $f(x)$ and $g(x)$ point in similar directions in a vicinity of $S$, so that the vectors in $H(x)$ point toward a regular domain irrespective of $\alpha$. In case (b) both vector fields point towards the switching domain, so that one can find a unique value of $\alpha$ such that $H(x)$ points in the direction of $S$ (in this case we say that the solution exhibits stable sliding motion in $S$ ). Uniqueness of solutions is lost when both vector fields point away from the switching domain, in which case solutions starting at $S$ cannot be uniquely defined and any small perturbation will drive $x$ away from $S$ (referred to as an unstable sliding motion).

Next we identify the above scenarios for the PA system defined in (20). To that end we define the sets

$$
\begin{aligned}
& \Omega_{f}^{-}=\left\{x \in D_{f} \cup S: f_{2}(x)-\beta f_{1}(x) \leq 0\right\}, \\
& \Omega_{f}^{+}=\left\{x \in D_{f} \cup S: f_{2}(x)-\beta f_{1}(x)>0\right\}, \\
& \Omega_{g}^{-}=\left\{x \in D_{g} \cup S: g_{2}(x)-\beta g_{1}(x) \leq 0\right\}, \\
& \Omega_{g}^{+}=\left\{x \in D_{g} \cup S: g_{2}(x)-\beta g_{1}(x)>0\right\} .
\end{aligned}
$$

Lemma 4.1 [Crossings between regular domains] The solutions of (20) cross from $D_{g}$ to $D_{f}$ in the set

$$
L_{g f}=\Omega_{f}^{+} \cap \Omega_{g}^{+} \subseteq S,
$$

and cross from $D_{f}$ to $D_{g}$ in the set

$$
L_{f g}=\Omega_{f}^{-} \cap \Omega_{g}^{-} \subseteq S,
$$

Proof: We only prove the first case (i.e. that if the solution reaches $L_{g f}$ defined in (24), then it crosses from $D_{g}$ to $D_{f}$ ); the converse case follows analogously. From Filippov's construction, the vector field in $S$ has the form

$$
\dot{x}=\left[\begin{array}{l}
\alpha f_{1}(x)+(1-\alpha) g_{1}(x) \\
\alpha f_{2}(x)+(1-\alpha) g_{2}(x)
\end{array}\right]
$$

with $0 \leq \alpha \leq 1$. By the definition of $\Omega_{f}^{+}$and $\Omega_{g}^{+}$, for any point $x \in L_{g f}$ we have that

$$
\begin{aligned}
\alpha f_{2}(x)+(1-\alpha) g_{2}(x) & >\alpha \beta f_{1}(x)+(1-\alpha) \beta g_{1}(x), \\
& >\beta\left(\alpha f_{1}(x)+(1-\alpha) g_{1}(x)\right),
\end{aligned}
$$

which implies that the vector field points to $D_{f}$ for any $0 \leq \alpha \leq 1$. In other words, the set $H(x)$ in 22 is fully contained in the regular domain $D_{f}$ and hence the trajectory crosses the switching domain.

Lemma 4.2 [Stable sliding motion] The solutions of (20) exhibit stable sliding motion in the set

$$
L_{s}=\Omega_{f}^{-} \cap \Omega_{g}^{+} \subseteq S
$$


Proof: We first prove by contradiction that for all $x\left(t_{0}\right) \in L_{s}$ the vector fields are such that $x(t)$ cannot leave the switching domain in an interval $\left(t_{0}, t_{0}+\Delta\right]$ . Define the absolutely continuous function

$$
\begin{gathered}
z:\left[t_{0}, t_{0}+\Delta\right] \rightarrow \mathbb{R}, \\
z(t)=x_{2}(t)-\beta x_{1}(t) .
\end{gathered}
$$

Suppose that there exists $\Delta>0$ such that $z(t)>0$ for $t \in\left(t_{0}, t_{0}+\Delta\right]$. If $x\left(t_{0}\right) \in S$ we have that $z\left(t_{0}\right)=0$, so by continuity it must be that $\dot{z}>0$ for $t \in\left(t_{0}, t_{0}+\Delta_{0}\right]$ and some $0<\Delta_{0} \leq \Delta$. In addition, from the definition of the PA system in $(20)$, if $z(t)>0$ then $\dot{x}=f(x)$ for $t \in\left(t_{0}, t_{0}+\Delta_{0}\right]$ and so

$$
\dot{z}=f_{2}(x)-\beta f_{1}(x), \text { for } t \in\left(t_{0}, t_{0}+\Delta_{0}\right] .
$$

However, the right-hand side of $(30)$ is continuous in $t$, and when $x\left(t_{0}\right) \in L_{s}$ it follows that $\dot{z} \leq 0$ for $t \in\left(t_{0}, t_{0}+\Delta_{0}\right]$, which is a contradiction. The converse argument can be used to show that $z(t)<0$ for $t \in\left(t_{0}, t_{0}+\Delta\right]$ leads to a contradiction. We thus conclude that $z(t)=0$ for $t \in\left[t_{0}, t_{0}+\Delta\right]$, and so $x(t) \in L_{s}$ for $t \in\left[t_{0}, t_{0}+\Delta\right]$.

The proof follows by checking that the vector fields for $x \in L_{s}$ are compatible with Filippov's construction, see [7]. If there is sliding motion in $L_{s}$, then there exists $\Delta>0$ such that

$$
\dot{z}=0, \text { for } t \in\left[t_{0}, t_{0}+\Delta\right]
$$

Since $x(t)$ must be a solution in Filippov's sense for $t \in\left[t_{0}, t_{0}+\Delta\right]$, then there must exist $0 \leq \alpha \leq 1$ such that

$$
\dot{x}=\alpha f(x)+(1-\alpha) g(x), \text { for } t \in\left[t_{0}, t_{0}+\Delta\right]
$$

Combining (31) and $(32)$ we get

$$
\begin{aligned}
0 & =\dot{x}_{2}-\beta \dot{x}_{1}, \\
& =\alpha f_{2}+(1-\alpha) g_{2}-\beta\left(\alpha f_{1}+(1-\alpha) g_{1}\right), \\
& =\alpha\left(f_{2}-\beta f_{1}\right)+(1-\alpha)\left(g_{2}-\beta g_{1}\right), \text { for } t \in\left[t_{0}, t_{0}+\Delta\right] .
\end{aligned}
$$

Solving for $\alpha$ in 33 gives

$$
\alpha(x)=\frac{g_{2}(x)-\beta g_{1}(x)}{\left(g_{2}(x)-\beta g_{1}(x)\right)-\left(f_{2}(x)-\beta f_{1}(x)\right)},
$$

For $x \in L_{s}$ it holds that $\left(f_{2}-\beta f_{1}\right) \leq 0$ and $\left(g_{2}-\beta g_{1}\right)>0$, therefore $\alpha(x)$ is unique for all $x \in L_{s}$ and satisfies $0 \leq \alpha(x) \leq 1$.

Lemma 4.3 [Unstable sliding motion] The solutions of 20 cannot be uniquely defined in the set

$$
L_{\bar{s}}=\Omega_{f}^{+} \cap \Omega_{g}^{-} \subseteq S
$$

Proof: Consider the function $z(t)$ defined in (29). As opposed to the proof of Lemma 4.2 in this case it can be shown that for $x\left(t_{0}\right) \in L_{\bar{s}}$ both $z(t)>0$ and $z(t)<0$ for $t \in\left(t_{0}, t_{0}+\Delta\right]$ are possible solutions. Note that another possible solution can be defined by picking $\alpha$ as in 34 so that $x(t)$ slides along $S$. 


\subsection{Geometric representation}

The results of last section define a partition of the switching domain into regions for crossings and sliding motions that has a convenient geometric representation. An alternative definition of the sets in 23 can be constructed as follows: define the normal vector of $S$ as $\eta^{\perp}=\left[\begin{array}{ll}-\beta & 1\end{array}\right]^{T}$, then $x \in \Omega_{f}^{-}$if and only if $\left\langle f(x), \eta^{\perp}\right\rangle \leq 0$, which after substitution of $f(x)=h^{f}-\Gamma x$ becomes

$$
\left\langle\Gamma\left(x-\phi^{f}\right), \eta^{\perp}\right\rangle \geq 0 .
$$

Analogous definitions can be constructed for the other sets in $(23)$. The boundary between $\Omega_{f}^{-}$and $\Omega_{f}^{+}$is the half-line

$$
C_{f}=\left\{x \in \mathbb{R}_{\geq 0}^{2}:\left\langle\Gamma\left(x-\phi^{f}\right), \eta^{\perp}\right\rangle=0\right\},
$$

whereas the boundary between $\Omega_{g}^{-}$and $\Omega_{g}^{+}$is

$$
C_{g}=\left\{x \in \mathbb{R}_{\geq 0}^{2}:\left\langle\Gamma\left(x-\phi^{g}\right), \eta^{\perp}\right\rangle=0\right\} .
$$

The focal points satisfy $\phi^{f} \in C_{f}$ and $\phi^{g} \in C_{g}$, respectively. Moreover, $C_{f}$ and $C_{g}$ are parallel and thus the sets $L_{s}$ and $L_{\bar{s}}$ correspond to the intersection between $S$ and the band generated by $C_{f}$ and $C_{g}$. Figure 3 illustrates this idea; a necessary condition for sliding motion (stable or unstable) is that the band between $C_{f}$ and $C_{g}$ intersects $S$ in $\mathbb{R}_{\geq 0}^{2}$. Moreover, inspection of Figure 3 suggests that the partition precludes the existence of stable and unstable sliding motion in the same switching domain (i.e. at least one of the sets $L_{s}$ and $L_{\bar{s}}$ is empty). Next we provide a geometric condition for characterizing these scenarios.

Lemma 4.4 Let $\vartheta_{1}=\angle\left(\Gamma\left(\phi^{f}-\phi^{g}\right), \eta^{\perp}\right)$. If

$$
\vartheta_{1} \in\left[-\pi,-\frac{\pi}{2}\right) \cup\left(\frac{\pi}{2}, \pi\right],
$$

unstable sliding motion cannot exist in $S$. Conversely, if

$$
\vartheta_{1} \in\left(-\frac{\pi}{2}, \frac{\pi}{2}\right)
$$

stable sliding motion cannot exist in $S$.

Proof: $C_{f}$ and $C_{g}$ intercept the vertical axis of $\mathbb{R}_{>0}^{2}$ at $p^{f}=\gamma_{2}^{-1}\left\langle\Gamma \phi^{f}, \eta^{\perp}\right\rangle$ and $p^{g}=\gamma_{2}^{-1}\left\langle\Gamma \phi^{g}, \eta^{\perp}\right\rangle$, respectively. Whether $L_{s}=\emptyset$ or $L_{\bar{s}}=\emptyset$ depends on $\operatorname{sgn}\left(p^{f}-p^{g}\right)=\operatorname{sgn}\left\langle\Gamma\left(\phi^{f}-\phi^{g}\right), \eta^{\perp}\right\rangle$ (see Figure 3), which leads to the angle conditions in $39-40$.

\subsection{Equilibria}

The focal points are locally stable equilibria of (20) provided that they belong to their respective cone, i.e. $\phi^{f} \in D_{f}$ or $\phi^{g} \in D_{g}$ (stability follows from $\gamma_{1}, \gamma_{2}>0$ ). However, as suggested by Figure 3 , it is possible that the trajectories reach an equilibrium that lies in the switching domain. This kind of equilibrium is sometimes termed singular equilibrium [2] and must be understood in Filippov's 

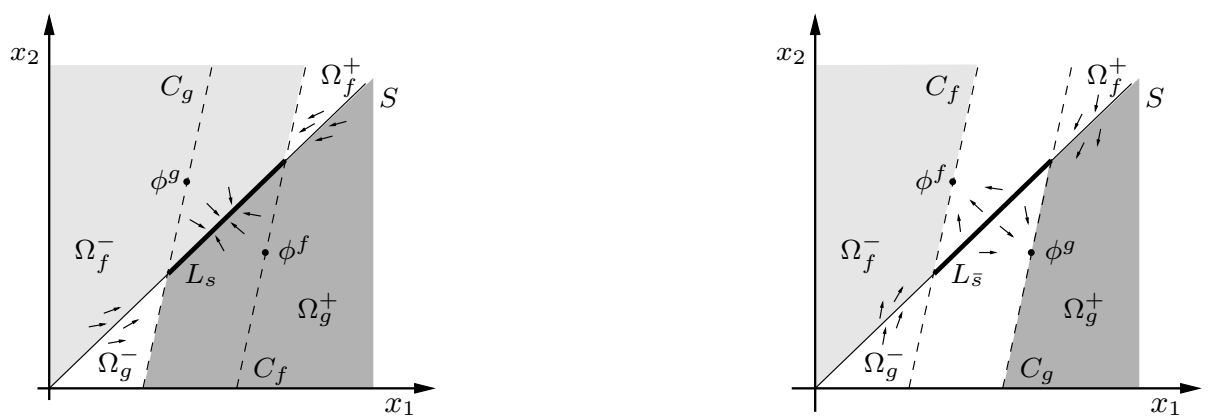

Figure 3: Switching domain with stable (left) and unstable (right) sliding motion.

sense (that is, at a singular equilibrium the convex hull $H(x)$ in 22 contains the origin). Depending on the location of the focal points, a singular equilibrium may exist in $L_{s}$; we characterize this statement more precisely in the next lemma.

Lemma 4.5 [Singular equilibrium] Assume that $L_{s} \neq \emptyset$; let $L_{\phi}$ be the line containing $\phi^{f}$ and $\phi^{g}$ and $\vartheta_{2}=\angle\left(\phi^{f}-\phi^{g}, \eta^{\perp}\right)$. The point

$$
\phi_{s}=L_{s} \cap L_{\phi},
$$

is a singular equilibrium of 20). Moreover, if

$$
\vartheta_{2} \in\left[-\pi,-\frac{\pi}{2}\right) \cup\left(\frac{\pi}{2}, \pi\right]
$$

then $\phi_{s}$ is locally stable, and if

$$
\vartheta_{2} \in\left(-\frac{\pi}{2}, \frac{\pi}{2}\right),
$$

then $\phi_{s}$ is unstable.

Proof: The proof follows by looking at the form of the vector field along $S$ when solutions are defined with Filippov's method. When $x \in L_{s}$ the solution satisfies

$$
\dot{x}=\alpha f(x)+(1-\alpha) g(x),
$$

with $\alpha=\alpha(x)$ given in (34). Substituting $\alpha(x)$ in (44) we get

$$
\dot{x}=\frac{A_{f g}(x) \eta}{\left(g_{2}(x)-\beta g_{1}(x)\right)-\left(f_{2}(x)-\beta f_{1}(x)\right)},
$$

where $A_{f g}(x)$ is given by

$$
A_{f g}(x)=\gamma_{1} \gamma_{2}\left\{x^{T} P\left(\phi^{f}-\phi^{g}\right)+\phi^{f^{T}} P \phi^{g}\right\},
$$

with $P=\left[\begin{array}{cc}0 & 1 \\ -1 & 0\end{array}\right]$ so that $x^{T} P x=0$ for all $x \in \mathbb{R}^{2}$. A point $\phi_{s} \in L_{s}$ is a singular equilibrium of $(20)$ if it satisfies $A_{f g}\left(\phi_{s}\right)=0$. The equation $A_{f g}(x)=0$ is satisfied by both focal points, i.e. $A_{f g}\left(\phi^{f}\right)=A_{f g}\left(\phi^{g}\right)=0$, and so the curve

$$
L_{\phi}=\left\{x \in \mathbb{R}_{\geq 0}^{2}: A_{f g}(x)=0\right\},
$$


is the line containing both focal points. We thus conclude that any singular equilibrium must be located at $\phi_{s}=L_{\phi} \cap L_{s}$. The stability of $\phi_{s}$ follows by examining the direction of the vector field in 45 . We know that

$$
\left(g_{2}(x)-\beta g_{1}(x)\right)-\left(f_{2}(x)-\beta f_{1}(x)\right)>0,
$$

for all $x \in L_{s}$, and hence the direction of the right-hand side of 45 depends only on the sign of $A_{f g}(x)$ along $L_{s}$. The function $A_{f g}(x)$ evaluated along $L_{s}$ (i.e. when $x=x_{1} \cdot \eta$ ) defines a line

$$
\left.A_{f g}(x)\right|_{x \in L_{s}}=\gamma_{1} \gamma_{2}\left\{\left\langle\phi^{f}-\phi^{g}, \eta^{\perp}\right\rangle x_{1}+\phi^{f^{T}} P \phi^{g}\right\},
$$

with slope

$$
\left.\frac{\partial}{\partial x_{1}} A_{f g}(x)\right|_{x \in L_{s}}=\gamma_{1} \gamma_{2}\left\langle\phi^{f}-\phi^{g}, \eta^{\perp}\right\rangle .
$$

Note that the line in 49 is transversal to the line $L_{\phi}$ and they intersect at $\phi_{s}$ (because $A_{f g}\left(\phi_{s}\right)=0$ and $\left.\phi_{s} \in L_{s}\right)$. Therefore $A_{f g}(x)$ changes sign at $x=\phi_{s}$, so the local stability of $\phi_{s}$ depends on the sign of the slope in (50); namely

$$
\phi_{s} \text { is }\left\{\begin{array}{ll}
\text { stable } & \text { if }\left\langle\phi^{f}-\phi^{g}, \eta^{\perp}\right\rangle<0 \\
\text { unstable } & \text { if }\left\langle\phi^{f}-\phi^{g}, \eta^{\perp}\right\rangle>0
\end{array},\right.
$$

which are equivalent to the angle conditions in $421-(43)$ (note that $\left\langle\phi^{f}-\right.$ $\left.\phi^{g}, \eta^{\perp}\right\rangle \neq 0$ since $\phi^{f}, \phi^{g} \notin S$ by assumption).

The result in Lemma 4.5 provides a geometric condition to check the existence of singular equilibrium points for the PA system (20). These equilibria can be locally stable or unstable, a property that can be graphically checked with a simple angle condition; the stable and unstable cases are illustrated in Figure 4 (a) and Figure 4(b), respectively. The PA system can also lack a singular equilibrium, in which case $L_{s} \cap L_{\phi}=\emptyset$ and the solutions may exhibit sliding motion in $L_{s}$ but eventually escape to one of the regular domains; this scenario is shown in Figure 4(c).

(a)

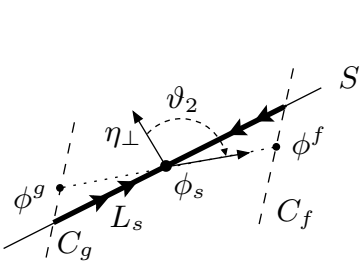

(b)

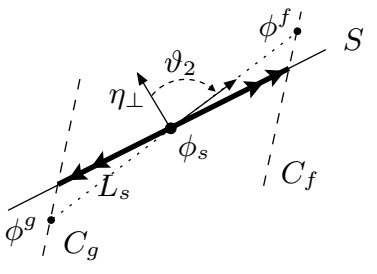

(c)

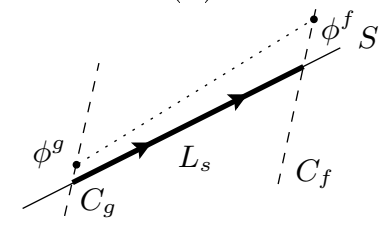

Figure 4: Singular equilibrium for the PA system in 20). 


\section{$5 \quad$ Equilibria of genetic control circuit}

In this section we return to the original problem of determining the metabolite and enzyme equilibria of the system in (1)-(2), after its reduction to a PA system defined in three conic domains, see $(15)$. If $\theta_{1}=\theta_{\ell}$, then the region $R_{1 \ell}$ is empty, and the equilibria can be obtained directly with the analysis in Section 4. In the case $\theta_{1} \neq \theta_{\ell}$, the idea is to use Lemmas 4.4 and 4.5 by splitting the three cone case into a pair of two cone problems: one for the switching domain $S_{1}$ with the focal points $\phi^{1}$ and $\phi^{1 \ell}$, and another one for $S_{\ell}$ with the focal points $\phi^{1 \ell}$ and $\phi^{\ell}$.

As discussed in Section 3.2 the location of the focal points depends on the regulatory logic in $(9)$, which is comprised in the functions $\sigma_{1}$ and $\sigma_{\ell}$. If $\theta_{1} \neq \theta_{\ell}$ and regardless of the particular regulatory logic, one pair of focal points will share the same vertical coordinate and another pair will share the horizontal coordinate. We can then use our results to show that the system can have one or two stable equilibria. Due to space constraints we only focus in the case of Figure 2, with $\theta_{1}<\theta_{\ell}$.

Lemma 5.1 [Equilibrium of genetic circuit] Consider the PA system in (15) with $\sigma_{1}=\sigma^{-}$and $\sigma_{\ell}=\sigma^{-}, \theta_{1}<\theta_{\ell}$, and the focal points located as in Figure 2. Then, (15) has only one equilibrium point $\phi_{s}^{1}$, which is singular, locally stable, and located at

$$
\phi_{s}^{1}=L_{\phi} \cap S_{1}
$$

where $L_{\phi}$ is the line containing $\phi^{1}$ and $\phi^{1 \ell}$.

Proof: In Figure 2 we see that the focal points lie outside their corresponding regular domains, so they cannot be equilibria of $(15)$. The only option then is to look for singular equilibria in $S_{1}$ or $S_{\ell}$. Denote the normal vectors to $S_{1}$ and $S_{\ell}$ as $\eta_{1}^{\perp}$ and $\eta_{\ell}^{\perp}$, respectively. We proceed by cases:

- $\phi^{f}=\phi^{1 \ell}$ and $\phi^{g}=\phi^{\ell}$ are aligned vertically, and hence

$$
-\frac{\pi}{2} \leq \angle\left(\Gamma\left(\phi^{1 \ell}-\phi^{\ell}\right), \eta_{\ell}^{\perp}\right) \leq 0
$$

for all $\gamma_{1}, \gamma_{2}>0$; from Lemma 4.4 (53) implies that $S_{\ell}$ cannot contain any stable sliding motion.

- $\phi^{f}=\phi^{1}$ and $\phi^{g}=\phi^{1 \ell}$ are aligned horizontally, and thus we have

$$
-\pi \leq \angle\left(\Gamma\left(\phi^{1}-\phi^{1 \ell}\right), \eta_{1}^{\perp}\right) \leq-\frac{\pi}{2},
$$

for all $\gamma_{1}, \gamma_{2}>0$; from Lemma $4.4(54)$ implies that $S_{1}$ can contain a region for stable sliding motion; the existence, location and stability of a singular equilibrium in $S_{1}$ follow directly from Lemma 4.5 and inspection of Figure 2

The equilibrium enzyme concentrations for the feedback system in (1)-(2) can be directly obtained with Lemma 5.1. Assuming that $\theta_{1}<\theta_{\ell}$ and given 
that $\phi_{s}^{1} \in S_{1}$ is a singular equilibrium, we have $\bar{s}_{\ell-1}=\theta_{1}$ and hence (see Figure 2 .

$$
\bar{e}_{1}=\frac{1}{\beta_{1}} \bar{e}_{\ell}, \quad \bar{e}_{\ell}=\frac{\kappa_{\ell}^{0}+\kappa_{\ell}^{1}}{\gamma_{\ell}} .
$$

The remaining equilibrium enzymes can be computed from $\sqrt{2}$ as

$$
\bar{e}_{i}=\frac{\kappa_{i}^{0}+\kappa_{i}^{1} \sigma_{i}\left(\theta_{1}, \theta_{i}\right)}{\gamma_{i}}, i \neq 1
$$

where the threshold are assumed to satisfy $\theta_{i} \neq \theta_{1}$ for $i \neq 1$. The equilibrium metabolites can be computed by solving the algebraic equation (cf. (7))

$$
g_{i}\left(\bar{s}_{i-1}\right)=g_{1}\left(s_{0}\right) \frac{\bar{e}_{1}}{\bar{e}_{i}}, i>1, i \neq \ell \text {. }
$$

As an illustrative example, in Figure 5 we plot the $\left(e_{1}, e_{\ell}\right)$ phase plane and the metabolite trajectories for the system in (1)-(2), together with its QSS approximation in $(9)$. The simulations in Figure 5 were obtained for a system of length $n=2$ with $\sigma_{1}=\sigma^{-}, \sigma_{\ell}=\sigma^{-}$, substrate $s_{0}=1$, Michaelis-Menten kinetics $\left(g_{i}=k_{\text {cat } i} s_{i-1} /\left(K_{\mathrm{m} i}+s_{i-1}\right)\right.$, with $k_{\text {cat } i}=2 \cdot 10^{2}$ and $K_{\mathrm{m} i}=5$, $i=1,2,3)$, regulatory parameters $\theta_{i}=\{0.5,1,1.5\}, \kappa_{i}^{0}=\{0.08,0.15,0.3\}$, $\kappa_{i}^{1}=10 \kappa_{i}^{0}$, and degradation rates $\gamma_{i}=\{0.25,0.9,0.8\}$.

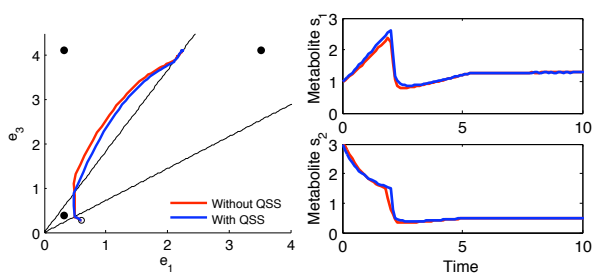

Figure 5: Numerical example; effect of QSS approximation.

\section{Discussion \& outlook}

The analysis carried out in this paper shows how genetic regulation of enzyme activity can generate different metabolic equilibria. This mechanism can control metabolic adaptations to environmental changes and is based on the interaction between metabolic species and transcription factors which modulate enzyme biosynthesis.

Key elements in our analysis are the use of a PA model for gene regulation and exploiting the time scale separation between metabolic and genetic dynamics. Applying a quasi steady state approximation ultimately permits to study the $n$-species gene circuit by a 2 -dimensional PA system defined in conic domains. The algebraic equations in (7) will be a good approximation of the metabolite trajectories provided that the time constants of the enzyme kinetics are much smaller than protein half-lifes, which in turn are inversely proportional to their degradation rates $\gamma_{i}$. In addition, enzyme saturation may invalidate the 
QSS approximation when a positive solution of (7) fails to exist. This issue has been briefly mentioned, and will be addressed in an upcoming paper.

The analysis of the reduced PA system with Filippov's method [5] allows the derivation of geometric conditions on the protein expression and degradation rates to identify the behavior of solutions at the switching domain. We have obtained two angle conditions to check the existence of stable or unstable sliding motion (Lemma 4.4), and the existence of singular equilibria at the switching domains (Lemma 4.5). By combining these two results we can characterize the equilibria for a given combination of gene regulatory parameters. Because of length constraints we have limited the analysis to the setup of Lemma 5.1 (see Figure 22, but the procedure can be applied to any combination of activation or repression feedback loops.

\section{References}

[1] Uri Alon. An introduction to systems biology: design principles of biological circuits. Chapman \& Hall/CRC, 2006.

[2] Richard Casey, Hidde de Jong, and Jean-Luc Gouzé. Piecewise-linear models of genetic regulatory networks: equilibria and their stability. J. Math. Biol., 52:27-56, 2006.

[3] M. Chaves, E.D. Sontag, and R. Albert. Methods of robustness analysis for boolean models of gene control networks. IEE Proc. Syst. Biol., 153:154$167,2006$.

[4] A. Cornish-Bowden. Fundamentals of Enzyme Kinetics. Portland Press, third edition, 2004.

[5] Aleksei F. Filippov. Differential equations with discontinuous righthandsides. Kluwer Academics Publishers, 1988.

[6] Leon Glass and Stuart A. Kauffman. The logical analysis of continuous, nonlinear biochemical control networks. J. Theor. Biol., 39:103-129, 1973.

[7] Jean-Luc Gouzé and Tewfik Sari. A class of piecewise linear differential equations arising in biological models. Dynamical Systems, 17(4):299-316, 2002.

[8] L.C.G.J.M. Habets and Jan van Schuppen. A control problem for affine dynamical systems on a full-dimensional polytope. Automatica, 40:21-35, 2004.

[9] Oliver Kotte, Judith B Zaugg, and Matthias Heinemann. Bacterial adaptation through distributed sensing of metabolic fluxes. Mol Syst Biol, 6(355), 2010 .

[10] D. Ropers, H. de Jong, M. Page, D. Schneider, and J. Geiselmann. Qualitative simulation of the carbon starvation response in Escherichia coli. BioSystems, 84:124-152, 2006. 
Centre de recherche INRIA Sophia Antipolis - Méditerranée 2004, route des Lucioles - BP 93 - 06902 Sophia Antipolis Cedex (France)

Centre de recherche INRIA Bordeaux - Sud Ouest : Domaine Universitaire - 351, cours de la Libération - 33405 Talence Cedex Centre de recherche INRIA Grenoble - Rhône-Alpes : 655, avenue de l'Europe - 38334 Montbonnot Saint-Ismier

Centre de recherche INRIA Lille - Nord Europe : Parc Scientifique de la Haute Borne - 40, avenue Halley - 59650 Villeneuve d'Ascq Centre de recherche INRIA Nancy - Grand Est : LORIA, Technopôle de Nancy-Brabois - Campus scientifique 615, rue du Jardin Botanique - BP 101 - 54602 Villers-lès-Nancy Cedex

Centre de recherche INRIA Paris - Rocquencourt : Domaine de Voluceau - Rocquencourt - BP 105 - 78153 Le Chesnay Cedex

Centre de recherche INRIA Rennes - Bretagne Atlantique : IRISA, Campus universitaire de Beaulieu - 35042 Rennes Cedex Centre de recherche INRIA Saclay - Île-de-France : Parc Orsay Université - ZAC des Vignes : 4, rue Jacques Monod - 91893 Orsay Cedex 